\title{
Radiation from surface of two-dimensional communication sheet
}

\author{
Akihito Noda ${ }^{\text {a) }}$, Akimasa Okada, and Hiroyuki Shinoda \\ Graduate School of Frontier Sciences, The University of Tokyo, \\ 5-1-5 Kashiwanoha, Kashiwa-shi, Chiba 277-8561, Japan \\ a)Akihito_Noda@ipc.i.u-tokyo.ac.jp
}

\begin{abstract}
Two-dimensional communication (2DC) is a wireless communication system using a waveguide sheet as a radio frequency (RF) signal transmission medium. This paper proposes a theoretical analysis model of RF radiation from a $2 \mathrm{DC}$ sheet surface. Previous works supposed that only a non-radiative guided mode propagates along a 2DC sheet and that radiation is due only to open ends of the sheet or to impedance discontinuities around the sheet edges. The proposed model based on field equivalence principle clarifies that the sheet guided mode propagating in a finite-size sheet inherently generates radiation fields even if no edge radiation nor impedance discontinuity are involved.
\end{abstract}

Keywords: two-dimensional communication, waveguide sheet, microwave, radiation

Classification: Antennas and Propagation

\section{References}

[1] H. Shinoda, Y. Makino, N. Yamahira, and H. Itai, "Surface sensor network using inductive signal transmission layer," Proc. $4^{\text {th }}$ INSS, Braunschweig, Germany, pp. 201-206, June 2007. DOI:10.1109/INSS.2007.4297420

[2] A. Noda and H. Shinoda, "Wireless LAN on 2-D communication tiles using ultra-wideband as an alternative spectrum resource," Proc. ICUWB 2015, Montreal, Canada, pp. 1-6, October 2015. DOI:10.1109/ICUWB.2015.7324530

[3] A. Noda and H. Shinoda, "Selective wireless power transmission through highQ flat waveguide-ring resonator on 2-D waveguide sheet," IEEE Trans. Microw. Theory Techn., vol. 59, no. 8, pp. 2158-2167, August 2011. DOI:10.1109/ TMTT.2011.2156425

[4] N. Yamahira, Y. Makino, H. Itai, and H. Shinoda, "Proximity connection in two-dimensional signal transmission," Proc. SICE-ICASE International Joint Conference 2006, Busan, Korea, pp. 2735-2740, October 2006. DOI:10.1109/ SICE.2006.315199

[5] N. Kobayashi, H. Fukuda, and T. Tsukagoshi, "Challenging EMC problems on two-dimensional communication systems," Proc. $7^{\text {th }}$ INSS, Kassel, Germany, pp. 130-137, June 2010. DOI:10.1109/INSS.2010.5574063

[6] H. Fukuda, N. Kobayashi, K. Nakase, and T. Tsukagoshi, "Methods for suppressing edge radiation from two-dimensional communication sheet," Proc. $8^{\text {th }}$ INSS, Penghu, Taiwan, pp. 1-4, June 2011. 
[7] R. F. Harrington, Time-Harmonic Electromagnetic Fields (Reissue), ch. 5-7, pp. 228-230, Wiley-IEEE Press, New York, 2001.

\section{Introduction}

Two-dimensional communication (2DC) is a radio system using a waveguide sheet as a radio frequency $(\mathrm{RF})$ signal transmission medium [1]. A special coupler that transmits/receives RF signals into/from the sheet is embedded in 2DC transceivers. The most dominant signal path is not an aerial radiation but consists of a near-field coupling between the sheet and coupler and of the sheet guided mode. Therefore unwanted RF radiation from the system can be reduced and 2DC enables lowemission ultra-wideband (UWB) high-speed communication [2] and safe wireless power transmission [3]. For appropriate design minimizing RF radiation from the sheet, an analysis model of the radiation is desired.

In this paper we propose a theoretical analysis model of RF radiation from a 2DC sheet surface. The proposed model clarifies that the guided mode propagating in a finite-size sheet inherently generates radiation fields. This fact has been missing in the analysis of 2DC sheet in previous works since the first proposal of 2DC using a waveguide sheet [4].

In previous works, Kobayashi et al. pointed out that radiation from the open edges of the sheet is a dominant factor and it is reduced by shorting out the edges [5]. Fukuda et al. reported that the short-ended sheet involves radiation due to the impedance discontinuity at the boundary between the mesh region and the shielded region and it can be reduced by gradual impedance transition between those regions [6]. These causes of radiation from the sheet are illustrated in Fig. 1.

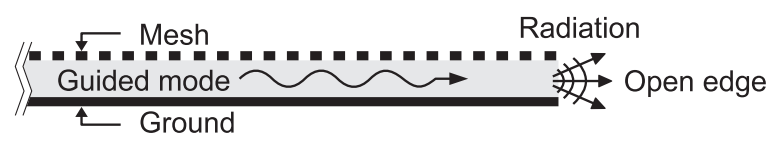

(a)

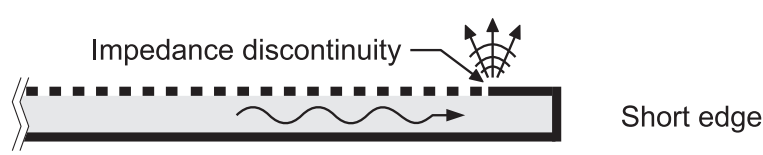

(b)

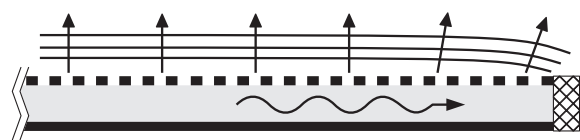

Perfectly absorbing boundary

(c)

Fig. 1. Cross sectional view around a 2DC sheet edge. Two significant causes of sheet radiation were addressed in previous works: (a) open edge and (b) impedance discontinuity of short-ended sheet. (c) This paper addresses another one, radiation from the whole mesh surface. 
impedance discontinuities or open edges. The radiation due to the traveling wave in one direction is a basis for composing the radiation field from actual 2DC sheet surface. Since the electromagnetic (EM) field in an actual 2DC sheet can be expanded in the plane wave basis, the radiation caused by these waves can also be expanded by the same manner.

The total radiation from the sheet in an actual 2DC system will be expressed as the superposition of radiations from the surface, edges, impedance discontinuities and any other scattering objects on the sheet. Thus, to estimate the total radiation, the analysis model presented in this paper should be combined with other models expressing radiations due to other causes.

In Section 2, the analysis model will be presented. The radiation fields calculated by the model and computed by a simulation software will be presented in Section 3. The paper will be concluded in Section 4.

\section{Theoretical analysis model}

For a concise discussion of the issue, in this section we will derive only a far-zone approximation of the magnetic field radiated from a 2DC sheet surface. Since we are interested in the electromagnetic interference between the 2DC sheet and other radio systems apart from the sheet due to the radiation from the sheet, the far-zone approximation will be derived. Suppose that only the lowest-order transverse magnetic (TM) guided mode [1] propagates in the 2DC sheet at the frequency of interest. The sheet lies in the $x y$-plane and the $+z$-direction is from the ground plane to the mesh plane. In this paper we consider the guided mode propagating in the $+x$-direction, and suppose that the EM field is invariant in the $y$-direction. This is a two-dimensional (2-D) problem in the $x z$-plane. The magnetic field vector lies in the $y$-axis and electric field vector lies in the $x z$-plane.

The guided mode comprises an evanescent field above the sheet surface as shown in Fig. 2(a). Non-zero components of the evanescent field is $E_{x}, E_{z}$, and $H_{y}$. From the view point of radiation field calculation, the EM fields traveling inside the sheet, $E_{s x}, E_{s z}$ and $H_{s y}$, do not directly contribute to the radiation and its only contribution is to generate the evanescent field above the sheet. Therefore the guided mode is modeled as the equivalent current and charge sources on the perfect electric conductor (PEC) ground plane to generate the identical evanescent field above the plane, as shown in Fig. 2(b). The sheet thickness $h$ is neglected, because it is significantly smaller than the distance between the sheet and any far-field observation points.

The original EM field intensity, $\boldsymbol{E}=\left(E_{x}, 0, E_{z}\right)$ and $\boldsymbol{H}=\left(0, H_{y}, 0\right)$, in the vicinity of the ground plane is equivalent to a magnetic current density $\boldsymbol{M}=$ $\left(M_{x}, M_{y}, M_{z}\right)$, an electric current density $\boldsymbol{J}=\left(J_{x}, J_{y}, J_{z}\right)$ and an electric charge density $q$ on the ground as expressed as follows:

$$
\begin{gathered}
\boldsymbol{M}=-\hat{\boldsymbol{z}} \times \boldsymbol{E}=\left(0,-E_{x}, 0\right) \\
\boldsymbol{J}=\hat{\boldsymbol{z}} \times \boldsymbol{H}=\left(-H_{y}, 0,0\right) \\
q=\varepsilon_{0} \hat{\boldsymbol{z}} \cdot \boldsymbol{E}=\varepsilon_{0} E_{z},
\end{gathered}
$$




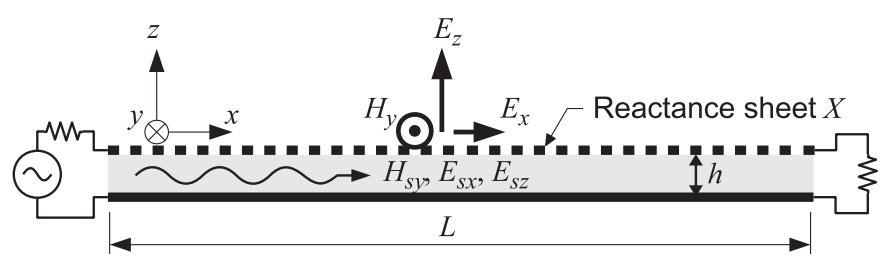

(a)

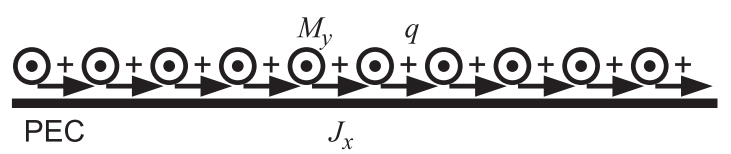

(b)

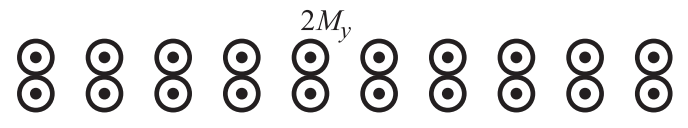

(c)

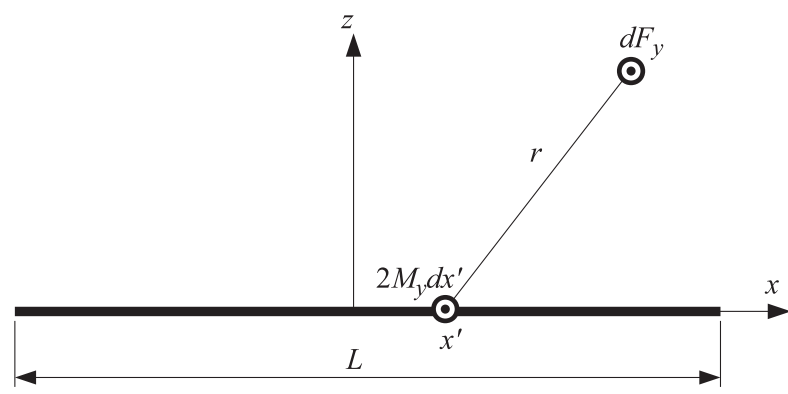

(d)

Fig. 2. Illustration of the proposed analysis model. The original guided mode propagation illustrated in (a) can be interpreted as an equivalent sources $M_{y}, J_{x}$ and $q$ on the ground plane, as shown in (b), where the 2DC sheet thickness $h \ll r$ is neglected and the sheet structure is removed. From the image theory, $J_{x}$ and $q$ are canceled by their images and $M_{y}$ is doubled, therefore the model reduces to one shown in (c). The magnetic current sheet $M_{y}$ spans the entire length of the 2DC sheet, $L$, and generates electric vector potential $F_{y}$ in the free-space as shown in (d).

where $\hat{\boldsymbol{z}}$ represents a unit vector in the $+z$-direction. Since these sources are on the PEC plane, $\boldsymbol{J}$ and $q$ are respectively canceled by their images. On the other hand, $M_{y}$ is doubled by its image. Thus, the evanescent field on the sheet is equivalent to the magnetic current sheet with the density $2 M_{y}=-2 E_{x}$ in the free space, as shown in Fig. 2(c).

In this model, by using a Green's function $G(r)$, the electric vector potential $\boldsymbol{F}=\left(0, F_{y}, 0\right)$ is related to the magnetic current source $\boldsymbol{M}$ as

$$
F_{y}=2 \int_{-L / 2}^{L / 2} G(r) M_{y}\left(x^{\prime}\right) d x^{\prime},
$$

where $r$ represents the distance between the source at $\left(x^{\prime}, 0\right)$ and the observation point at $(x, z)$ in the $x z$-plane as shown in Fig. 2(d):

$$
r=\sqrt{\left(x-x^{\prime}\right)^{2}+z^{2}}
$$


Note that Eq. (4) is not valid for the back side of the sheet $(z<0)$, because it is fictitious image field. To derive the field invariant in $y$-direction, the following 2-D free-space Green's function is used [7]:

$$
G(r)=-\frac{j \varepsilon_{0}}{4} H_{0}^{(2)}\left(k_{0} r\right) .
$$

For far-zone approximation, the Hankel function of the second kind, $H_{0}^{(2)}\left(k_{0} r\right)$, can be expressed as the following asymptotic formula.

$$
G(r) \approx-\frac{j \varepsilon_{0}}{4} \sqrt{\frac{j 2}{\pi k_{0} r}} e^{-j k_{0} r}=-\frac{\varepsilon_{0}}{\sqrt{j 8 \pi k_{0} r}} e^{-j k_{0} r}
$$

The magnetic field $\boldsymbol{H}$ is calculated from $\boldsymbol{F}$ as

$$
\boldsymbol{H}=-j \omega\left(\boldsymbol{F}+\frac{1}{k_{0}^{2}} \nabla \nabla \cdot \boldsymbol{F}\right),
$$

where $\nabla \cdot \boldsymbol{F}=\partial F_{y} / \partial y=0$ for our 2-D model, therefore

$$
H_{y}(x, z)=-j \omega F_{y}(x, z) .
$$

Substituting Eqs. (1), (4) and (7) into Eq. (9) and we obtain

$$
H_{y}(x, z) \approx-\sqrt{j} \frac{\omega \varepsilon_{0}}{\sqrt{2 \pi k_{0}}} \int_{-L / 2}^{L / 2} E_{x}\left(x^{\prime}\right) \frac{e^{-j k_{0} r}}{\sqrt{r}} d x^{\prime} .
$$

The original evanescent electric field $E_{x}$ of the guided mode wave traveling in the $+x$-direction with the wavenumber $k_{S}$ is expressed as

$$
E_{x}\left(x^{\prime}\right)=A e^{-j k_{S} x^{\prime}}
$$

where $A$ represents the amplitude, assumed to be constant in $-L / 2 \leq x \leq L / 2$ by neglecting the attenuation of the guided wave due to the radiation. By substituting Eq. (11) into Eq. (10), we finally obtain

$$
H_{y}(x, z) \approx-\sqrt{j} \frac{\omega \varepsilon_{0} A}{\sqrt{2 \pi k_{0}}} \int_{-L / 2}^{L / 2} \frac{e^{-j k_{0} r} e^{-j k_{S} x^{\prime}}}{\sqrt{r}} d x^{\prime} .
$$

\section{Numerical simulation}

The radiated magnetic field patterns numerically computed with the proposed model and obtained by an EM simulator, CST Microwave Studio, are shown in Figs. 3(a) and (b), respectively. Only the far-zone, $z \geq 60 \mathrm{~mm}$, corresponding to $k_{0} z \gtrsim 10$, is plotted. Note that the approximation in the proposed model does not assume $r \gg L$ but only assumes $k_{0} r \gg 1$. Both of them were calculated for $1-\mathrm{W} / \mathrm{m}$ power density (power per unit width of the sheet) of signal input into the sheet at $f=8 \mathrm{GHz}$. The discrepancy between the results of the proposed calculation and of the CST simulation is shown as the ratio between them in Fig. 3(c). Before calculating the ratio, the simple moving average filter with one-wavelength $(37.5 \mathrm{~mm})$ square window was applied to the both results, because we are not interested in the discrepancy due to the slight difference in the wavelength between the proposed model and the EM simulation. Note that broader ripples found in Fig. 3(b) and (c) are generated by reflected waves due to the imperfection of the absorbing boundary of EM simulation model. 


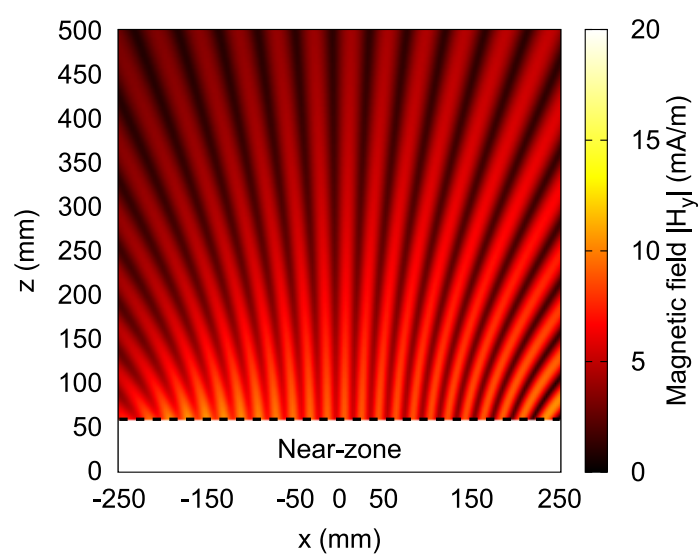

(a)

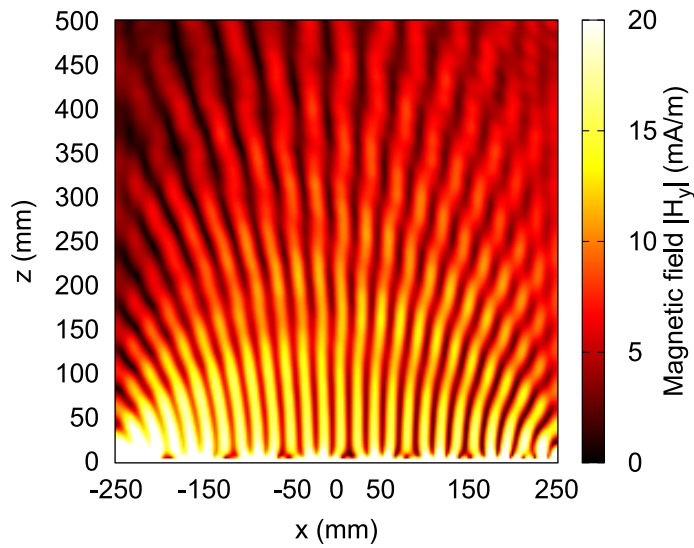

(b)

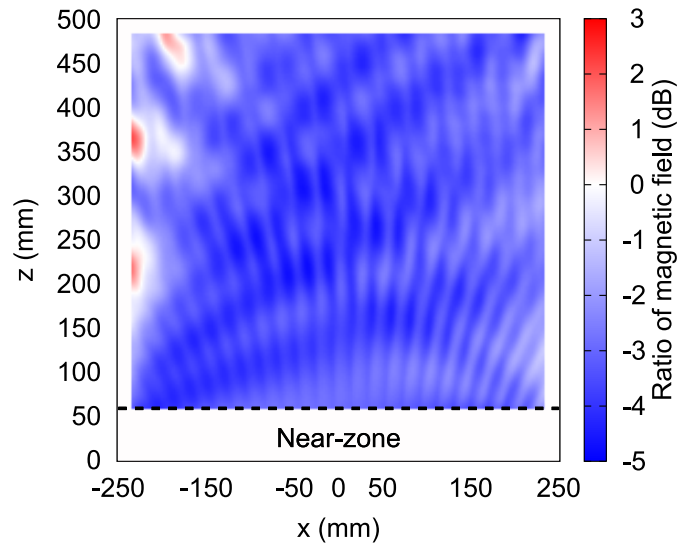

(c)

Fig. 3. Magnetic field amplitude radiated from 2DC sheet surface.

(a) Field calculated by the theoretical model and (b) simulated by CST Microwave Studio. Note that the theoretical model neglects the near-field and CST simulates complete near- and far-fields. (c) The ratio of the proposed model's magnetic field to the simulated one. Moving average filter was applied to the both magnetic field data before calculating the ratio.

The simulation model of CST Microwave Studio is a quasi-2-D model, i.e., the model width in the $y$-axis is one period of the periodic mesh structure and the boundary conditions at $\pm y$-boundaries are perfect magnetic conductor to force $H_{x}=H_{z}=0$. The EM wave source is connected at the $-x$-end of the sheet. The 
$\pm x$ - and the $+z$-boundaries are set to be perfect absorbing boundaries. Thus, the model is equivalent to Fig. 2(a). The mesh pitch (period) is $4 \mathrm{~mm}$ and the conductor line width is $1 \mathrm{~mm}$, the same as the sheet used in [2]. From the simulation result, the effective sheet reactance of the mesh was determined as $X \approx 9.8 \Omega / \mathrm{sq}$.

For the above condition, the amplitude $A$ in Eq. (11) was determined as approximately $27 \mathrm{~V} / \mathrm{m}$. The wavenumbers of the plane wave in the free-space and of the sheet guided mode are respectively $k_{0}=2 \pi f / c_{0}$ and $k_{S} \approx 1.56 k_{0}$, where $c_{0}$ is the speed of light. $k_{S}$ was determined by the EM simulator.

The calculated magnetic field pattern well agrees to the simulated pattern, as shown in Fig. 3(a) and (b). It indicates that the proposed model well describes the cause of radiation. Their discrepancy evaluated with the moving-average filtered patterns was roughly $3 \mathrm{~dB}$ as shown in Fig. 3(c). Thus, the results demonstrate the validity of the proposed model.

\section{Conclusion}

This paper proposed a theoretical analysis model of RF radiation from a 2DC sheet surface. With the proposed model, the calculated radiation is not due to any openedges nor any impedance discontinuities, but to the guided mode propagating in a finite-size sheet. It has been missing in the analysis of $2 \mathrm{DC}$ sheet in the previous works.

Although the radiation was calculated for a traveling wave in only one direction, the total radiation from an actual 2DC sheet can be calculated by the same manner as plane wave expansion of fields in the sheet. Therefore, any schemes found to be effective to reduce radiation in the model will be also effective for actual 2DC sheet. As one of our future works, a low-emission 2DC sheet will be designed based on the analysis with the proposed model. Although the model derived in this paper is a 2-D model, it can be extended to the 3-D version by replacing the Green's function and integrating over entire 2DC sheet surface in Eq. (4).

\section{Acknowledgments}

This work was supported in part by the Strategic Information and Communications R\&D Promotion Programme (SCOPE) 155103003. 